\title{
Studien über den photographischen Entwicklungsprozeß II (Feinkornentwicklung)
}

\author{
Von W. Meidinger \\ Mitteilung aus der Physikalisch-Technischen Bundesanstalt in Braunschweig \\ (Z. Naturforschg. 6 a, 375-376 [1951]; eingegangen am 30. April 1951)
}

Herrn Prof. Dr. John Eggert zum 60. Geburtstag gewidmet

\begin{abstract}
Durch mikroskopische Beobachtung des Entwicklungsvorganges am einzelnen Halogensilberkorn der photographischen Emulsion wird die charakteristische Wirkungsweise eines (echten) Feinkorn-Entwicklers klargelegt.
\end{abstract}

$I^{n}$ n einer früheren Arbeit ${ }^{1}$ war gezeigt worden, daß bei der Entwicklung der Bromsilberkörner einer photographischen Schicht (Einkornschicht) die (mittlere) Zeit vom Eindringen des Entwicklers in die Schicht bis zum sichtbaren Einsetzen der Entwicklung (bei mikroskopischer Beobachtung im inaktiven Licht) und die daran sich anschließende (mittlere) Zeit bis zur Durchentwicklung (Reduktion) der Körner sich in sehr charakteristischer Art unterscheiden:

Bei einer unbelichteten Schicht sind nach einem bestimmten Zeitpunkt, etwa nach 5 Min., nur sehr wenige Körner, die sogenannten Schleierkörner, entwickelt und erst nach sehr langen Entwicklungszeiten setzen mehr und mehr Körner mit der Entwicklung ein, bis schließlich alle Körner zu schwarzem Silber reduziert sind. In der belichteten Schicht sind, wiederum nach etwa 5 Min., mit zunehmender Belichtung steigende Mengen von Körnern entwickelt, bis im Gebiet sehr starker Belichtung, im Solarisationsgebiet, die Anzahl der entwickelten Körner wieder abnimmt. Man kann dieses Verhalten der Bromsilberkörner einer photographischen Schicht, wie es Eg g e rt einmal sehr treffend ausdrückte, folgendermaßen charakterisieren: Die mittlere „Lebensdauer“ der Körner im Entwickler wird durch die Belichtung verringert.

Demgegenüber ist nun aber die mittlere Zeit vom Einsetzen der Entwicklung an bis zur völligen Durchreduktion der Körner einer Größenklasse stets von der Belichtung unabhängig bzw. konstant, sowohl bei den Schleierkörnern in der unbelichteten Schicht als auch bei normaler Belichtung oder im Solarisationsgebiet.

Bei geeigneten Versuchsbedingungen, (grobkörnige Emulsion; geeigneter, genügend schwacher Entwickler) erscheint das Einsetzen der Entwicklung am Korn ziemlich sprunghaft, so daß die Zeit bis zur An-

1 W. M e i ding e r, Physik. Z. 36, 312 [1935]. entwicklung einerseits und die Durchreduktionszeit andererseits recht scharf zu beobachten sind. So kann im Gebiet sehr schwacher Belichtung oder auch im Solarisationsgebiet ein Korn, z. B. etwa 15 Min., ohne jede sichtbare Veränderung im Blickfeld liegen, bis plötzlich ein schwarzer Punkt am Korn sichtbar wird und in einigen Sekunden die Durchentwicklung des Korns vollzogen ist.

Der Aufbau der Schwärzungskurve wird nach diesen Beobachtungen also durch die verschiedene „Lebensdauer“ der Körner und nicht etwa von verschiedenen Zeiten für die Durchreduktion der Körner bestimmt.

Es wurde nun auch schon weiter gefunden, daß die Durchreduktionszeiten bei normalen Rapidentwicklern durch Variation der Gesamtkonzentration der Entwicklerflüssigkeit, des Alkaligehaltes oder Bromidgehaltes des Entwicklers in gewissen Grenzen beeinflußbar sind (vgl. z. B. in ${ }^{1}$ die Abb. 5 bis 11). Nicht untersucht wurde damals der Einfluß verschiedener Entwicklersubstanzen. Es war daher von Interesse, in der erprobten Art zu untersuchen, ob man insbesondere bei (echten) Feinkornentwicklern charakteristische Unterschiede bei den Anentwicklungszeiten und Durchreduktionszeiten gegenüber Rapidentwicklern feststellen könnte.

$\mathrm{Zu}$ diesem Zweck wurden Einkornschichten mit einer möglichst grobkörnigen Emulsion² (V 95) hergestellt und stufenweise belichtet. Nach der Entwicklung, einerseits in einem normalen Rapidentwickler (Metol-Hydrochinon-Pottasche; 2 Min. bei $20^{\circ} \mathrm{C}$ ) und andererseits im Agfa-Atomal-Entwickler (4 Min.), wurde zunächst mit Essigsäure die weitere Entwicklung gestoppt und darauf fixiert, gewässert, getrocknet und mikrophotographiert. Ausschnitte der Mikro-

2 W. M e i d in g e r, Physik. Z. 38, 565 [1937]. 
photogramme sind in den Abb. $1 \mathrm{a}-\mathrm{c}^{*}$ für den normalen Rapidentwickler und in den Abb. $2 \mathrm{a}-\mathrm{c}$ für den Agfa-Atomal-Entwickler wiedergegeben.

Man erkennt - insbesondere bei den großen Körnern - deutlich, daß bei der Rapidentwicklung alle Körner durchreduziert sind, während in allen Fällen bei der Feinkornentwicklung die Körner nur erst zu einem Teil durchreduziert sind, obwohl hier doppelt so lange entwickelt wurde wie bei der Rapidentwicklung. Der Prozentsatz der anentwickelten Körner ist dagegen in beiden Fällen (hier allerdings nach verdoppelter Entwicklungszeit im Feinkornentwickler) etwa gleich, wie auch noch genauer mit Hilfe der früher ${ }^{1}$ angegebenen Zählmethode (hier jetzt bei gleichen Entwicklungszeiten) nachgewiesen werden konnte.

Mißt man nun weiter in der früher beschriebenen Weise $^{1}$ die mittlere Durchreduktionszeit der großen Körner, so findet man bei der Rapidentwicklung Werte zwischen 5 und 10 Sek., bei der Feinkornentwicklung Zeiten zwischen 5 und 10 Min., also ein Unterschied der Durchreduktionszeiten entsprechend dem Faktor $\sim 60$.

Oben war bereits erwähnt, daß man die Durchreduktionszeit bei einem Rapidentwickler ebenfalls z. B. durch Variation der Konzentration oder durch verschiedenen Alkaligehalt, beeinflussen kann, doch wird dann auch immer zugleich die "Lebensdauer“ der Körner verändert. Nach Abb. 6 in ${ }^{1}$ sind z. B. nach 5 Min. Entwicklungsdauer bei der

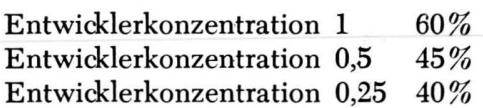

aller Körner anentwickelt.

* Abb. 1-4 s. Tafel S. 376 a.
Die charakteristische Wirkungsweise eines echten Feinkornentwicklers, wie ihn der Atomal-Entwickler darstellt, liegt offenbar darin, daß bei relativ zur Rapidentwicklung gleicher Anentwicklungsfähigkeit die Reduktionszeit der Körner stark verlängert wird, so daß nach einer entsprechend gemessenen Entwicklungsdauer nur erst kleine Teile der anentwickelten großen Körner zu Silber reduziert sind, diese also nach dem Fixieren in kleinere Silberteilchen aufgeteilt erscheinen, wodurch die Vergröberung der Körnigkeit durch die großen Körner weitgehend aufgehoben wird. Natürlich wird bei zu lang ausgedehnter Entwicklung diese Wirkung des Feinkornentwicklers rückgängig gemacht, da dann auch die großen Körner durchreduziert werden. Weiter ist nach diesen Beobachtungen offensichtlich, daß die F Finkornentwicklung gegenüber der Rapidentwicklung stets weniger Silber liefert, daß man also dementsprechend mit einer verringerten Schwärzung zu rechnen hat, was wiederum bedeutet, daß man eine Einbuße an Empfindlichkeit bei der Feinkornentwicklung in Kauf zu nehmen hat, wie in der Praxis allgemein bekannt ist. Einen Ausgleich in dieser Hinsicht, der sich vor allem bei den normalen, in der Praxis verwendeten dicken Schichten mit 30 bis 40 Kornlagen übereinander bemerkbar machen dürfte, kann man in der erhöhten Deckkraft (,photometrische Konstante"; $P=[\mathrm{Ag}] /$ Schwärzung) des feinerkörnigen Silbers gegenüber dem gröberkörnigen bei der Rapidentwicklung annehmen.

Schließlich zeigt die Gegenüberstellung der Abb. $3 \mathrm{a}-\mathrm{b}$ für den Rapidentwickler und $4 \mathrm{a}-\mathrm{b}$ für den Feinkornentwickler noch einmal sehr instruktiv die Wirkungsweise des Feinkornentwicklers hinsichtlich des praktischen Effektes durch die erzielte Feinkörnigkeit der fertigen fixierten Schicht. 
Abb. 1 a-c. Normale Rapidentwicklung (Metol-Hydrochinon-Pottasche, 2 Min. $\left.20^{\circ} \mathrm{C}\right)$.

Abb. 2 a-c. Feinkornentwicklung (AgfaAtomal, 4 Min. $20^{\circ} \mathrm{C}$ ).

Mikrophctogramme von Einkornschichten einer grobkörnigen Versuchsemulsion nach Entwicklung mit einem Rapidentwickler und einem Feinkornentwickler bei verschiedener Belichtung. (Entwicklung mit Essigsäure gestoppt; Schichten nicht fixiert, gewässert, getrocknet und mit $\lambda \sim 625 \mathrm{~m} \mu$ auf panchromatischer Platte mikrophotographiert; Abbildungsmaßstab etwa 1:800.
a) Belichtung 0 (Schleier)
b) Belichtung $10^{-3}$ Sek. $\quad \lambda=436 \mathrm{~m} u$.
c) Belichtung ca. $10^{-1}$ Sek.

Abb. $3 \mathrm{a}-$ b. Normale Rapidentwicklung (Metol-Hydrochinon-Pottasche, 2 Min. $\left.20^{\circ} \mathrm{C}\right)$.

Abb. $4 \mathrm{a}-\mathrm{b}$. Feinkornentwicklung (AgfaAtomal, $4 \mathrm{Min} .20^{\circ} \mathrm{C}$ ).

Mikrophotogramme von Einkornschichten einer grobkörnigen Versuchsemulsion nach Entwicklung mit einem normalen Rapidentwickler und einem Feinkornentwickler (Agfa-Atomal).

a) unfixierte Schicht, b) fixierte Schicht.
$1 \mathrm{a}$

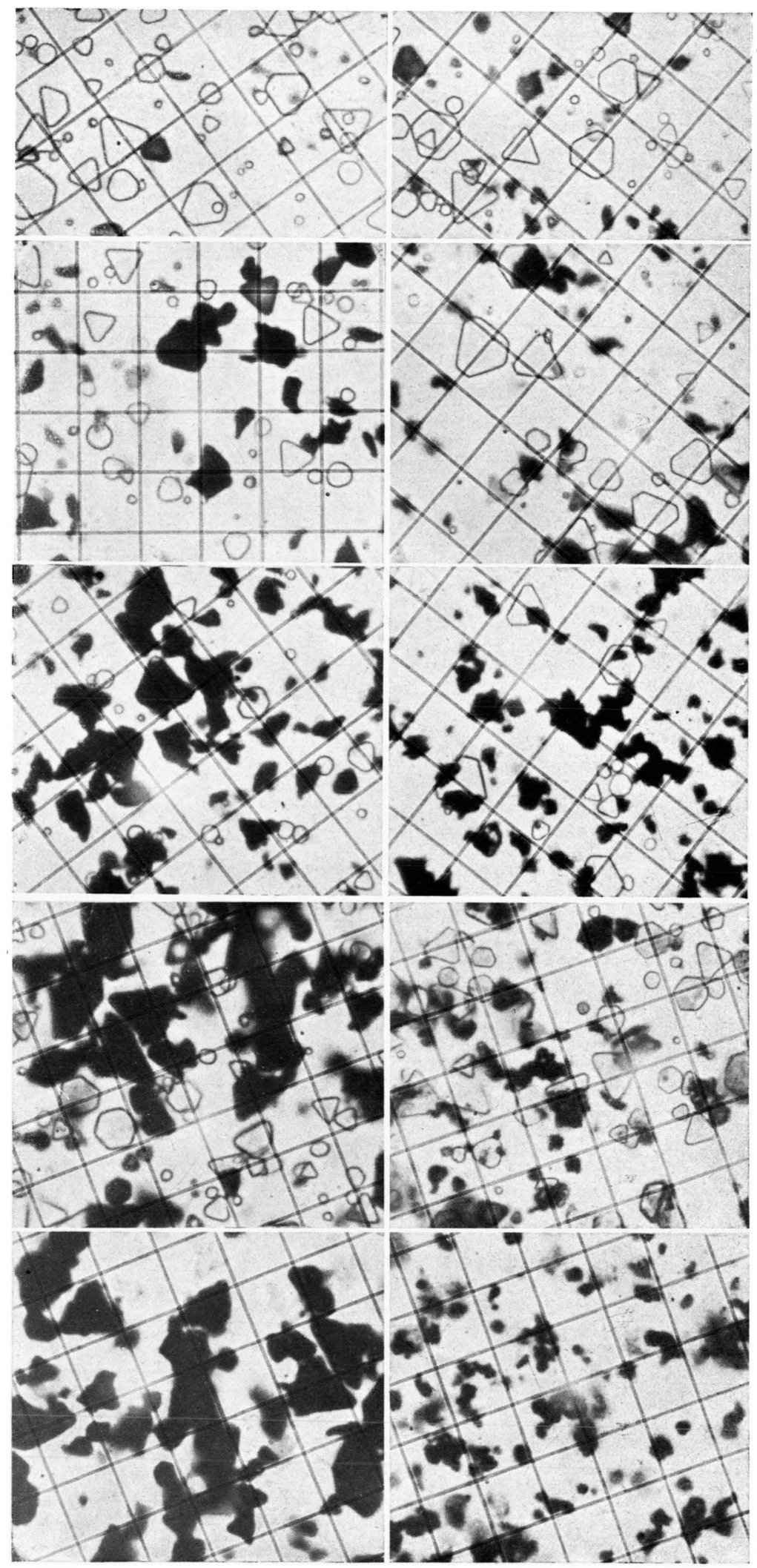


Physikalische Reifung einer Chlorsilber-Emulsion

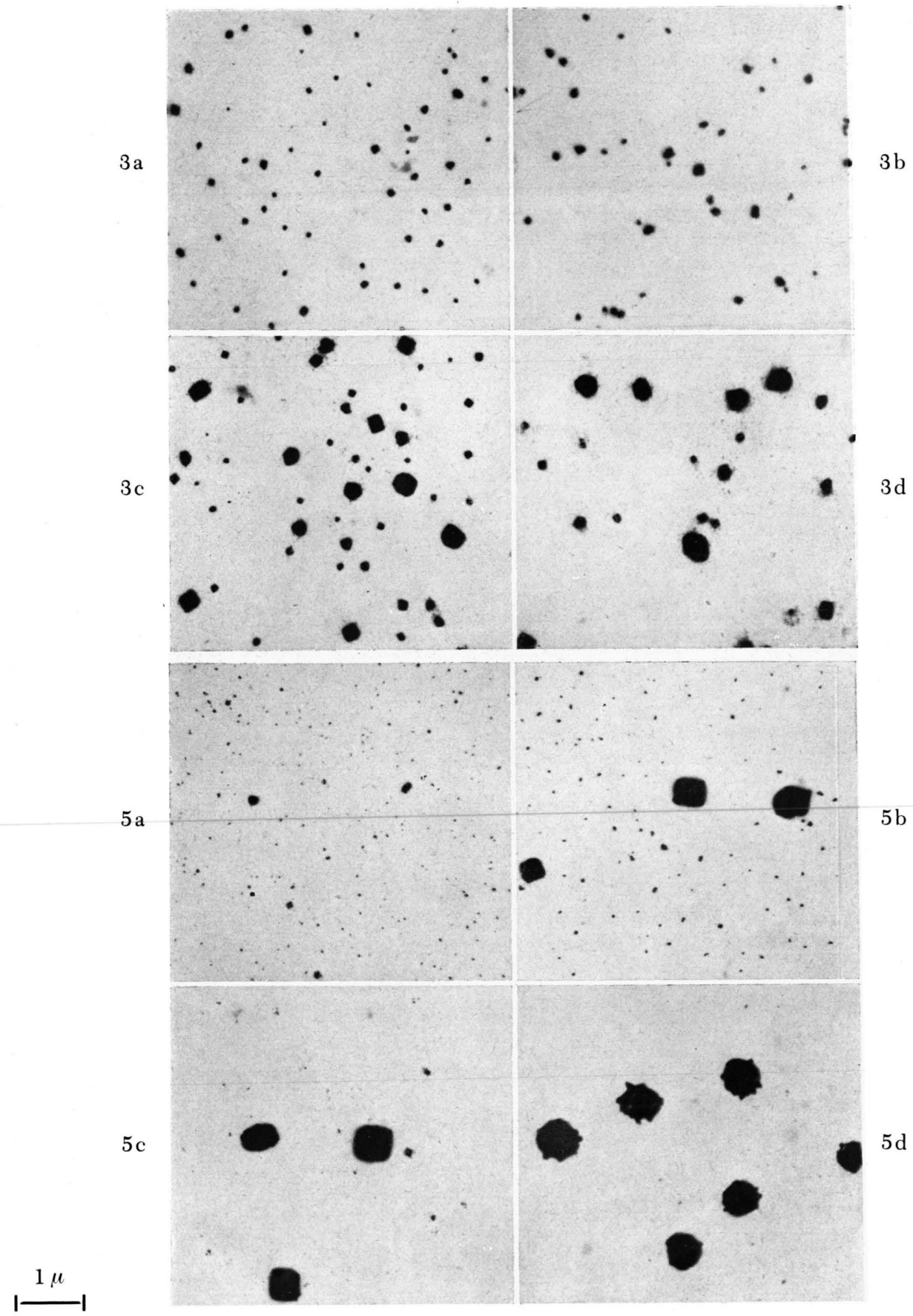

Abb. 3. Hochaktive Gelatine Winterthur No. 4199. Konzentration 0,5\%. Reifungsdauer: 3 a: 2 Min., 3 b: 4 Min., 3 c: 8 Min., $3 \mathrm{~d}$ : 16 Min.

Abb. 5. Hochaktive Gelatine Winterthur No. 4199. Konzentration 7\%. Reifungsdauer: 5 a: 2 Min., 5 b: 4 Min., 5 c: 8 Min., 5 d: 32 (!) Min. 